\title{
Editorial Preface and Acknowledgments
}

This book contains a new abridged translation of Galileo Galilei's Dialogue on the Two Chief World Systems, Ptolemaic and Copernican, together with a considerable amount of commentary designed to provide an introduction to the reading, understanding, appreciation, and criticism of this classic work. My commentary emphasizes the themes of critical reasoning and methodological reflection, and so it is also meant to provide a concrete introduction to critical thinking.

The translation has been made from the Italian text provided in volume 7 of the critical National Edition of Galileo's complete works edited by Antonio Favaro (1890-1909). I have, however, also consulted the text of the original edition published in 1632 ; this has been especially valuable in regard to matters of punctuation, which was almost completely reworked by Favaro; the original punctuation provides additional clues to the syntactical and logical structure of the text. Selected passages are identified in my translated text by the insertion of page numbers from that National Edition; these are designated by the numerals in square brackets interspersed within my translated text. Omitted passages are indicated by ellipses, and the notes give information about what is being left out. Occasionally there is an ellipsis within Galileo's own Italian text, and $I$ have noted the fact in the notes.

In making my translation, I have consulted and benefited from all previous English translations of Galileo's book, namely Webbe (I635), Salusbury (166I), Santillana (1953), and Drake (1967). However, I have 
tried to improve the accuracy of these without making my translation so literal as to lose readability; I have also aimed to facilitate comprehension rather than mere reading; and I have attempted to steer a middle course between an excessively free and easy-flowing translation and an excessively literal and hard-to-read one.

The selections have been made in accordance with the following principles. First, the focus is on the Copernican controversy; by this I do not mean exclusively or even primarily the issues pertaining to technical, mathematical, planetary astronomy, but rather those pertaining to cosmology, physics, methodology, and qualitative astronomy. Second, I include primarily passages which are explicit discussions of objections against or arguments in favor of the Copernican system. A third reason for inclusion stems from whether the passage is illustrative of an important philosophical or scientific idea, or informative about some significant historical incident. A fourth criterion is that each selection be a relatively self-contained unit which can be read and understood independently of the others. A fifth principle is that I want to maximize the variety of distinct scientific and philosophical issues discussed, and avoid excessive overlappings or near duplications. Finally, I have been sensitive to the requirement that the selections should not include too much of the original work, in order not to make the present book excessively and self-defeatingly long; the selections amount to about 38 percent of the original.

Galileo's text lacks any kind of subdivision other than the dedication, preface, and four chapters called Days. To help readers find their way, I have numbered my selections and given a title to each. These numbers and titles are placed in brackets in the text, in order to indicate that they are editorial additions. The titles are meant to be as descriptive and informative as possible while avoiding pedantry.

The Galilean text is preceded by an outline that subdivides each selection into a number of parts, in order to give a better overview of the content of each selection. These parts are listed in the order in which the respective topics arise in Galileo's discussion; but the correspondence is inexact, partly because the discussion in the dialogue does not proceed uniformly in a single direction, and partly because the various topics overlap to some extent. Nevertheless, the outline aims to provide a useful indication of the key points of any particular selection.

The notes are partly historical and partly philosophical; partly informative, partly interpretive, and partly critical. I have tended to avoid purely erudite commentary, but I have included scholarly points and 
bibliographical references which I felt were helpful. In regard to controversial issues, I have tried to indicate the nature of the issues rather than present just one side or my own resolution of the problem. My comments are meant to be relatively elementary and introductory, by which I mean that they involve simplifications and approximations, all of which are liable to further refinement and deeper analysis; however, I aim to distinguish simplifications from oversimplifications and approximations from distortions. In writing the notes, I have benefited from previous editions of Galileo's book and often adapted without acknowledgment the information they provide; therefore, here I should like to take this opportunity to explicitly acknowledge my debt to Strauss (1891), Santillana (1953), Pagnini (1964), Drake (1967), Sosio (1970), and Sexl and von Meyenn (I982). In those notes where my debt is more direct, I have explicitly acknowledged the fact, giving appropriate references and quotations from their works.

The introduction is meant to be a systematic and elementary exposition of the background historical information. It provides interpretive and narrative accounts of those developments in cultural, intellectual, and scientific history to which Galileo's book is directly connected, namely the Copernican Revolution, the Scientific Revolution, and the Galileo Affair. It also contains a preliminary sketch of those universal and perennial traits and activities of the human mind that are recorded in Galileo's book and make it a living document; these are the mental skills of critical reasoning, methodological reflection, and verbal rhetoric.

However, a systematic exposition of these concepts and their connection with critical thinking, together with a corresponding critical interpretation of the Dialogue, is to be found in the appendix rather than in the introduction. The appendix thus provides abstract articulations and textual illustrations of the guiding conceptual framework which I have both used in, and derived from, my critical reading of the book.

The commentary in the notes, introduction, and appendix explains most of the Galilean terms that are technical, semi-technical, archaic, personal names, or geographical names. It also explains special terms introduced by the present editor, for their explanations are usually given in the context where they are first used. However, there are a number of terms and concepts (both Galilean and editorial) which, for one reason or another, could not be efficiently or adequately explained in that commentary; they are explained in the glossary. The largest group of such terms are those Galilean words that are used in more than one selection; in these cases, if the explanation is not found in that commentary, the 
reader should look in the glossary. Another important group of glossary entries are those editorial terms that are especially ubiquitous in my commentary. In any case, the reader can consult the index for references to definitions and occurrences of all terms.

It should be noted that bibliographical references to Galileo's works in the notes are given by using his first name, by which he is generally referred to in English. This is done for the sake of uniformity since most such notes also use his first name in a nonbibliographical context. However, the bibliography lists his works in the standard manner under his last name.

Finally, in creating this work, I have benefited from many persons and institutions, and here I should like to express my acknowledgments to them.

I first conceived this book in 1980 at the time of the publication of my Galileo and the Art of Reasoning as a kind of sequel to that work; at that time I received the encouragement of Stillman Drake (Toronto). Later, after the publication of my Galileo Affair in 1989, I received the encouragement of Daniel Jones (National Endowment for the Humanities); he rekindled my interest, for I could then see myself doing with the text of the Dialogue something analogous to what my recent book had done with the documents of Galileo's trial. Then John L. Heilbron (Berkeley) responded favorably to the idea, and I am indebted to him for his constant support. Ron Naylor (Greenwich, England), William Shea (McGill), and Michael Segre (Munich) provided valuable encouragement and support at various stages of the project. Frederic L. Holmes (Yale) and Ernan McMullin (Notre Dame) made helpful comments on various progress reports. I also received valuable comments, suggestions, and constructive criticism from several scholars who read part or all of the book manuscript at various stages of its development: I. Bernard Cohen (Harvard), Allan Franklin (Colorado at Boulder), David Hill (Augustana College), Peter Machamer (Pittsburgh), Connie Missimer (independent scholar, Seattle), Albert Van Helden (Rice), and William A. Wallace (Maryland and Catholic University). My students Ioana Gal, John Ketchum, and Alan Rhoda provided invaluable assistance by being meticulous proofreaders and by playing the role of intelligent consumers and perceptive nonexpert readers.

Acknowledgments are also due to the National Endowment for the Humanities for a research grant (number RH-20980-9I) from 1992 to 1995, without whose support the required work would not have been done; this is especially true in the light of the fact that this grant was 
awarded by the special program in "guided studies of historically significant scientific writings from antiquity to the twentieth century" in the Humanities, Science, and Technology Category of the Division of Interpretive Research. At my own institution, the University of Nevada, Las Vegas, my gratitude goes to the following: the Sabbatical Leave Committee, for a sabbatical leave in fall 1994, during which this book was completed; and the College of Liberal Arts and the Department of Philosophy for a reduction in teaching load and assignment to the Center for Advanced Research in the spring semester of 1994. 
This page intentionally left blank 